Musées, Patrimoine et Culture scientifiques et techniques

175 | 2018

janvier-février 2018

\title{
"Le foot est un reflet de la vie sociale et contemporaine"
}

\section{Florent Molle et Samuel Cordier}

\section{QpenEdition \\ Journals}

Édition électronique

URL : http://journals.openedition.org/ocim/1899

DOI : $10.4000 /$ ocim.1899

ISSN : 2108-646X

Éditeur

OCIM

Édition imprimée

Date de publication : 1 janvier 2018

Pagination : 28-34

ISSN : 0994-1908

Référence électronique

Florent Molle et Samuel Cordier, «"Le foot est un reflet de la vie sociale et contemporaine" », La Lettre de I'OCIM [En ligne], 175 | 2018, mis en ligne le 01 juillet 2018, consulté le 19 avril 2019. URL : http:// journals.openedition.org/ocim/1899; DOI : 10.4000/ocim.1899

Ce document a été généré automatiquement le 19 avril 2019

Tous droits réservés 


\section{"Le foot est un reflet de la vie sociale et contemporaine"}

\section{Florent Molle et Samuel Cordier}

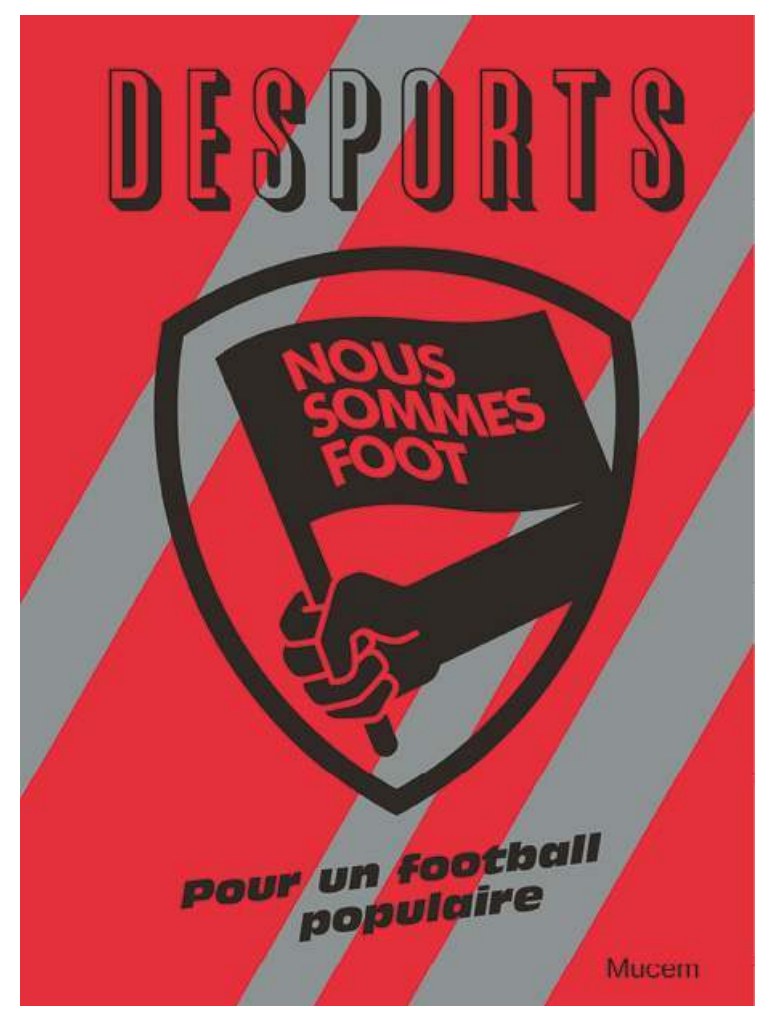

(c) Mucem

Comment avez vous eu l'idée de ce projet d'exposition ?

C'est Gilles Perez, le deuxième commissaire de l'exposition, qui a eu l'idée à l'été 2013. Il venait de réaliser un documentaire sur la fabrication du Mucem, qui s'appelle Naissance d'un musée, et a connu les équipes scientifiques à cette occasion. Par ailleurs, Zeev Gourarier, le directeur scientifique du musée souhaitait aborder le football dans 
une exposition. Du coup, après leur rencontre, Zeev a demandé à Gilles d'attendre qu'un conservateur soit intéressé par le projet pour construire un vrai projet d'exposition. Nous avons donc commencé à travailler ensemble en septembre 2013, deux mois après mon arrivée. Et nous avons ouvert l'exposition en octobre 2017. Cela fait quatre années de travail, avec quelques coupures.

Vous pensiez donc que le football pouvait avoir une place au musée?

Complétement oui. Travailler sur ce projet fut un plaisir du début jusqu'à la fin, mais il s'est aussi construit dans un contexte d'argumentation continue car ce n'est pas un sujet qui s'impose par nature, comme pourrait l'être une exposition biographique sur des ancêtres fondateurs comme Michel Leiris ou Georges Henri Rivière, des thèmes qui font peut-être plus l'unanimité dans le monde des musées et au Mucem. C'est compliqué de faire une exposition sur le football parce qu'il existe des préjugés, même ici en interne ; beaucoup avaient peur que nous soyons dans le cliché. Nous avons dû argumenter, mais justement c'est ce qui était intéressant, il a fallu tenir bon avec un sujet qui n'allait pas de soi. Nous avons travaillé avec un même objectif sur le long terme, pour réaliser une exposition pour tout le monde, rappeler le rôle citoyen du football, montrer comment il est un prisme pour comprendre la société.

Vous saviez dès le départ à quels publics se destinait cette exposition?

Non, nous ne nous sommes pas posés la question de nous adresser à un public en particulier, ce qui peut amener à forcer le trait, à être encore plus dans la caricature. D'emblée, nous avons voulu faire une exposition pour tout le monde. Nous étions convaincus que justement le football pouvait intéresser tout le monde. Pour nous, il n'existe pas de césure, de classes, sur l'intérêt lié au football. C'est au fur et à mesure, après avoir présenté le projet en interne, que nous avons constaté qu'il existait un préjugé qui était celui de dire que le public des musées ne vient pas au stade, et que le public des stades ne vient pas au musée. Même si c'est un préjugé qui a peut-être des bases réelles et a été vrai, nous nous sommes placés en opposition à celui-ci. Nous avons toujours dit qu'il fallait justement dépasser cette idée qui repose selon nous sur une fausse lecture de la société qui vise à éloigner deux classes de la société : les personnes qui regardent le football et celles qui visitent les musées.

Au début du projet, avez-vous visité d'autres expositions sur le football, ou plus généralement le sport?

Nous en avons beaucoup vu. Gilles en avait vu en Amérique du Sud et notamment le musée du football à San Paolo. Pour ma part, j'ai visité des musées en France avec le musée des Verts à Saint-Étienne, en Angleterre avec le National football Museum à Manchester ou le musée du club de Manchester United. Mais aussi en Espagne (FC Barcelona museum) ou en Italie (les musées de la Juventus de Turin et du Gran Torino). Et nous avons suivi les musées qui ouvraient en Europe, car il en existe aujourd'hui de plus en plus dans les clubs de football et de fédérations. Nous les avons visités, ce sont des musées qui présentent généralement un "hall of fame", des trophées, des maillots de grands joueurs, et des parcours muséographiques qui mettent en avant une épopée et une histoire glorieuse, en cachant parfois les points noirs de l'histoire du club ou qui pourraient faire défaut à cette image. Ce n'est pas du tout ce que nous avions envie de reproduire. Ce que souhaitions éviter, c'est reproduire cette marchandisation de cette culture football, comme nous pouvons le voir à Barcelone ou à Turin Nous nous sommes tournés vers d'autres types de musées pour chercher l'inspiration. Mais peu de musées à mon sens prennent le football au 
sérieux, c'est-à-dire comme un fait de société. Le musée national du Sport à Nice le fait très bien, mais propose des expositions plutôt historiques, dédiées à l'histoire de la Coupe du Monde ou de la Coupe d'Europe par exemple. Cela nous intéressait moins. En tant que musée de société, nous voulions vraiment nous intéresser à ce que nous disait le football sur le monde contemporain dans lequel on vit.

De l'esprit de quelles expositions vous sentiez-vous le plus proche?

L'exposition la plus marquante sur le sujet s'appelait Hors-jeu. Elle a été présentée en 2008 et 2009 au musée d'Ethnographie de Genève et a été réalisée par Rafaelle Poli et Christian Delécraz. Cette exposition a abordé le football comme un sport-spectacle dont l'étude permet de s'interroger sur la complexité de notre monde.

Et la trame s'est imposée comment? De manière évidente?

Ce découpage en trois parties - Passions, Engagements, Mercatos - est intervenu assez tôt. Dès le début, j'ai eu envie de parler des supporters et Gilles, de l'engagement politique à travers le football ; donc ces deux parties étaient à la base du projet. Ensuite, nous nous sommes demandés comment aborder l'économie. Parler d'une chose vécue par tous et, à première vue, immatérielle. Nous avons beaucoup travaillé pour savoir ce que nous allions exposer et nous avons pu collecter des objets au fur et à mesure de la réflexion. La partie sur l'économie était finalement la plus évidente, parce que tout le monde s'intéressait à ça au moment où nous produisions l'exposition. Et, en même temps, la plus difficile à penser muséographiquement parce qu'il existe très peu d'objets.

\section{Quels ont été les apports de votre comité scientifique?}

Nous avons mis en place un comité scientifique qui a regroupé des chercheurs et des joueurs. Il y avait notamment Rachid Mekhloufi, un ancien joueur de Saint-Étienne et de l'équipe nationale algérienne et Prédrag Pasiç, ancien joueur international bosnien qui a fondé une école multiethnique à Sarajevo et dont nous évoquons l'histoire dans l'exposition. Et puis des chercheurs qui avaient travaillé sur le football, comme Stéphane Mourlane, Christian Bromberger, Claude Boli ou Rafaelle Poli. Avec eux, nous avons réfléchi et leur avons proposé un projet qui mettait beaucoup en avant les supporters. C'est quelque chose qui me tenait à cœur. Gilles souhaitait aussi montrer le côté politique, car il avait réalisé un travail documentaire important sur les rebelles du football. Dans des contextes particuliers, comme dans celui du conflit israélo-palestinien ou de la guerre en ex-Yougoslavie, le football a été utilisé pour diffuser d'autres idées. Le comité scientifique nous a conseillés de surtout nous intéresser aux contradictions dans le football, comme des fils à tirer pour construire le propos. C'est important, car ces contradictions, celles d'un sport lié à un vrai business et en même temps basé sur des valeurs de solidarité, mettent en avant celles des sociétés contemporaines.

Vous avez également travaillé sur des récits.

En effet, le deuxième principe muséographique était de faire une exposition de récits. Nous avons trois parties - Passion, Engagement et Mercatos - ainsi qu'une introduction qui contient un vestiaire (où l'on se lave de ses idées préconçues sur le football) et une salle immersive. Et une conclusion, qui a pour titre "Prolongations", qui est là pour montrer les initiatives récentes pour un football plus citoyen. L'exposition de récits, c'est un concept issu du "musée de l'innocence" du romancier Orhan Pamuk ${ }^{1}$. C'est un petit musée, à Istanbul, qui part de son livre Le musée de 
l'innocence. Dans ce musée, Oran Pamuk raconte une histoire. Pour lui, nous ne sommes plus dans un monde qui a besoin d'épopées et de grandes histoires nationales, mais d'histoires intimes et de récits pour pouvoir le comprendre. Avec Nous sommes Foot, nous plongeons le visiteur dans des contextes particuliers, parfois guerriers, avec des témoignages et des récits qui permettent au visiteur de l'exposition de se projeter dans ces histoires. Par exemple, je pense que nous pouvons comprendre le conflit israélo-palestinien à travers le récit de la vie quotidienne de la footballeuse Honey Thaljieh. Nous essayons de créer une interaction entre une vitrine qui ne parle pas et un visiteur qui ne parle pas non plus, mais comprend. Les bouts de vies se répondent, se comprennent et permettent des croisements sociaux.

Les travaux de musées d'ethnographie vous ont également inspirés?

Le manifeste du musée d'Ethnographie de Neuchâtel, rédigé par Marc-Olivier Gonseth, Jacques Hainard et Roland Kaerh fut aussi une source d'inspiration. De manière plus large, l'ouvrage Cent ans sur la colline de Saint Nicolas dans lequel les partis pris muséographiques de chaque exposition du musée d'Ethnographie de Neuchâtel sont finement recensés, m'a donné beaucoup d'outils théoriques ou pratiques pour travailler sur le parcours. L'approche muséographique du musée d'Ethnographie de Neuchâtel a aussi permis d'amener de l'humour dans l'exposition. Nous avons pris la liberté de tourner en dérision certains aspects ou d'ironiser sur certaines pratiques, en plaçant par exemple une photographie de Maradona avec un maillot "no drugs" ou une compilation des plus belles simulations du football moderne. Il aurait peut être fallu plus d'humour dans l'exposition, mais c'était important car, en France, nous n'avons pas l'habitude de rire dans nos musées.

\section{Quels choix avez-vous faits pour la muséographie?}

Nous avons travaillé avec un collectif d'artistes espagnol qui s'appelle Democracia. Dès 2016, le collectif a proposé un projet articulé autour de l'idée d'un grand couloir qui distribue toutes les salles de l'exposition. Ils sont partis de l'idée de reprendre une architecture brutaliste, un peu comme à l'intérieur des premiers stades qui existaient en Europe, avec des ensembles architecturaux répétitifs. Nous cherchons à plonger le visiteur sous des gradins, avec des grands couloirs, éclairés par des néons très froids, comme ceux que l'on emprunte dans le stade pour chercher sa place. Nous avons aussi utilisé le grillage, comme un élément de la scénographie, qui rappelle ceux qui se situent parfois entre les supporters et le stade. Tout cela participe à la création d'une ambiance froide, industrielle, bétonnée, très proche de celle des stades d'aujourd'hui et assez éloignée de l'ambiance "fausse pelouse et filet de but" qui est généralement utilisée dans les expositions qui traitent du football. Le graphisme a aussi été réfléchi selon ce principe, avec de grands numéros et de grandes lettres, comme dans le stade, pour nous situer dans l'espace. Dans le stade, notre ticket nous indique notre place - bloc $\mathrm{B}$, porte 4 , place 65 - ici, ce sont des grands numéros qui indiquent chaque partie de l'exposition. Avec les étudiants du département son et image de Aix-Marseille Université, le Satis, nous avons pu créer un espace immersif au début de l'exposition. Les ambiances sonores de cet espace ont été réalisées à partir de sons collectés dans les vestiaires avant les matchs et dans des stades.

Vous souhaitiez également créer un espace pour échanger.

Nous avions envie qu'une agora existe dans l'exposition, pour créer un lieu de discussion et de débats. L'idée était de dire que le football est un reflet de la vie sociale et contemporaine. C'était un parti pris muséographique, nous ne voulions pas 
asséner de vérités et, parfois, pouvoir poser des questions. Par exemple, dans la partie religion, figure la question : "est ce que le football est une religion ou un opium du peuple ?".

Le nombre de sections de l'exposition, et son temps de visite, ce sont des clins d'œil ?

Bien sûr. Nous sommes dans un stade, c'est une exposition qui se visite en 90 minutes, découpée en onze parties... nous avons un peu tiré le fil, mais il s'agit plutôt de communication. Le sous-texte est lié au fait de dire que le stade est une métaphore de la société, au sein de laquelle nous cherchons notre place.

Seule la partie "hooligans" est traitée de manière différente.

En effet, pour cette partie, nous avons voulu faire une distinction de manière muséographique entre la culture ultras et le monde hooligan. Le hooliganisme est formellement mis en cage, avec une porte pour indiquer au visiteur que s'il souhaite faire une impasse sur cette partie et cette violence, il peut le faire, comme le spectateur peut refuser la violence. Pour passer par cette pièce, il faut ouvrir la porte, faire un choix.

Hans van der Meer, Marseille, Montredon, 2004. Centre national des arts plastiques

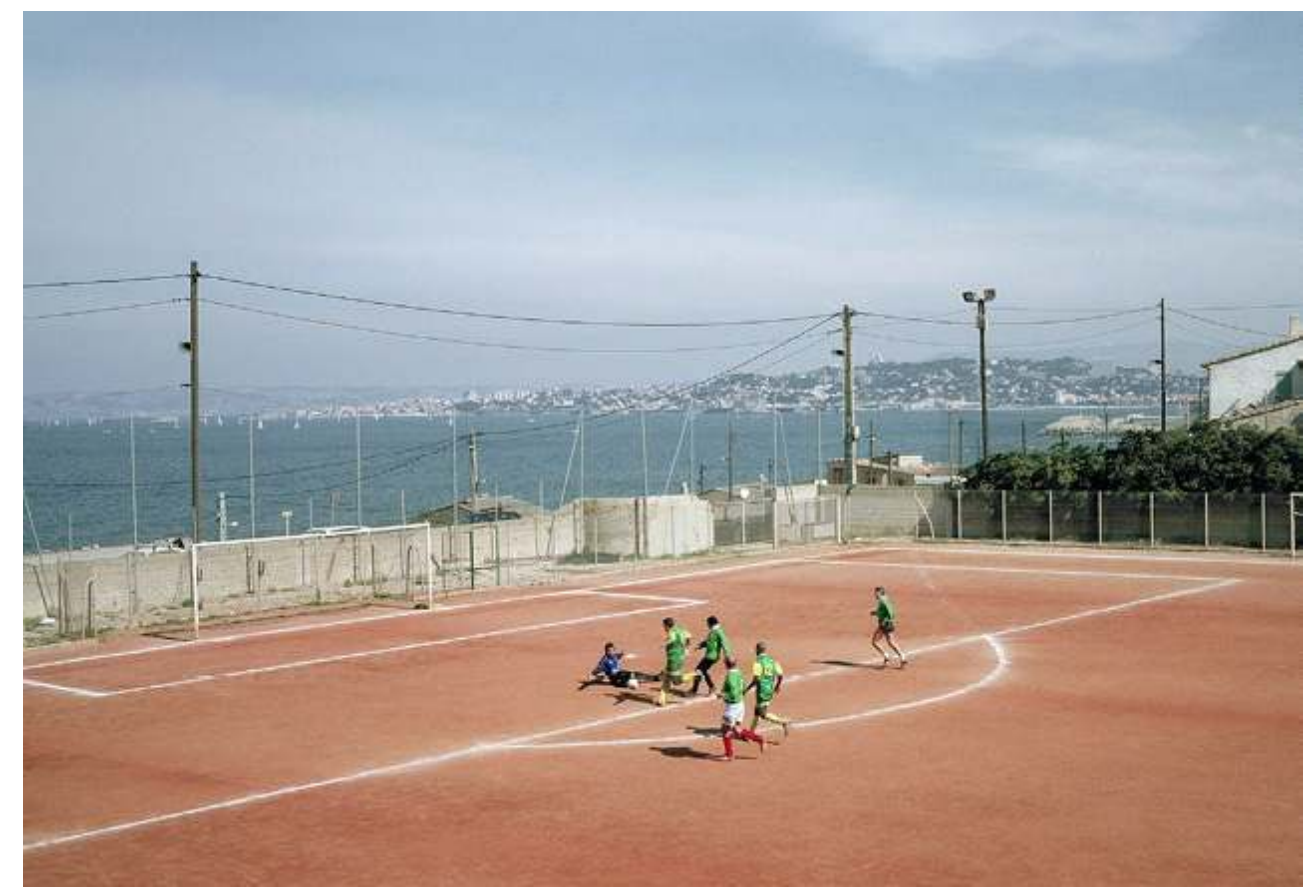

(c) Hans van der Meer / Hollandse Hootge, 2015 
OM - Olympique Lyonnais, Stade Vélodrome, Marseille, France, 16 août 2002, Lionel Briot

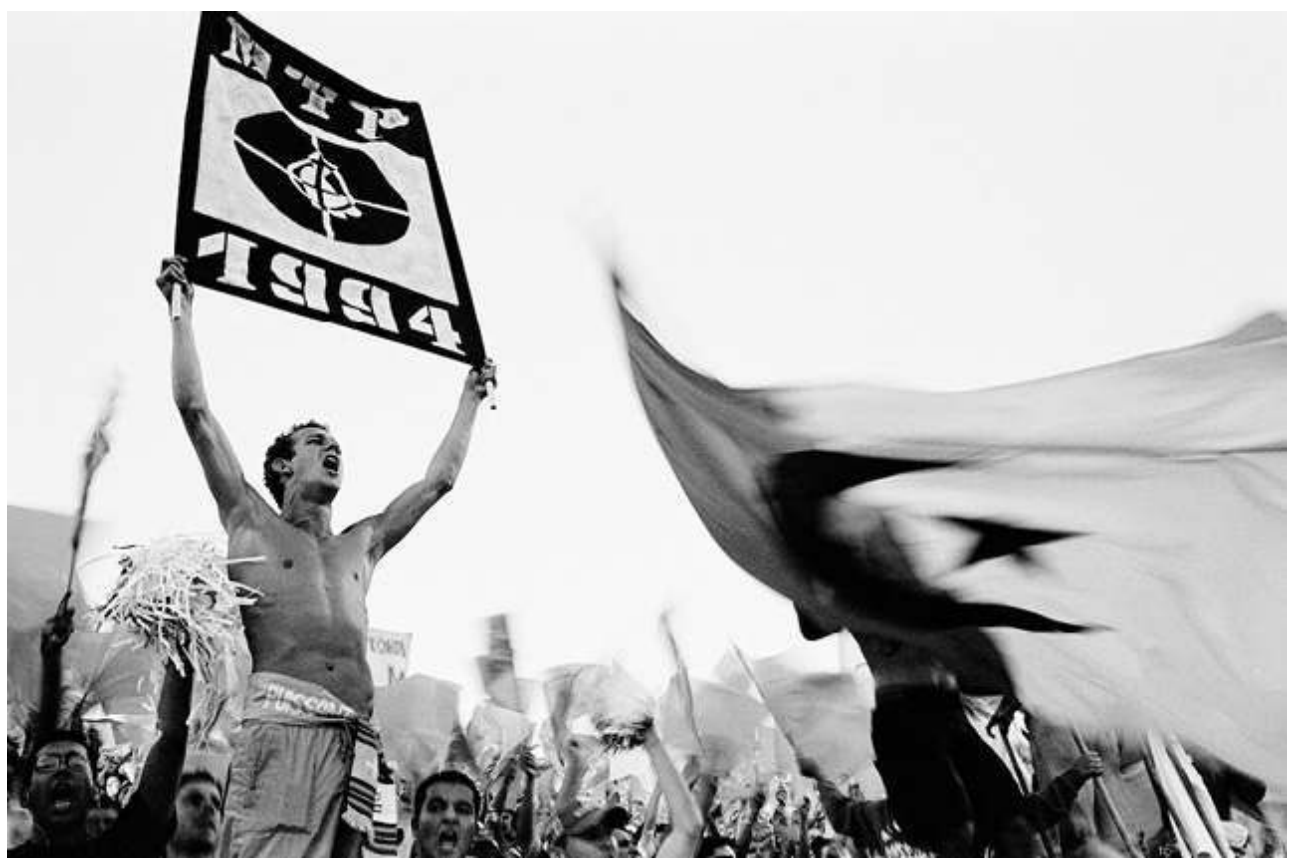

(c) ADAGP, Paris, 2018

Claude Lévêque, Hooligan, 2006

kamel mennour, Paris

Claude Lévêque

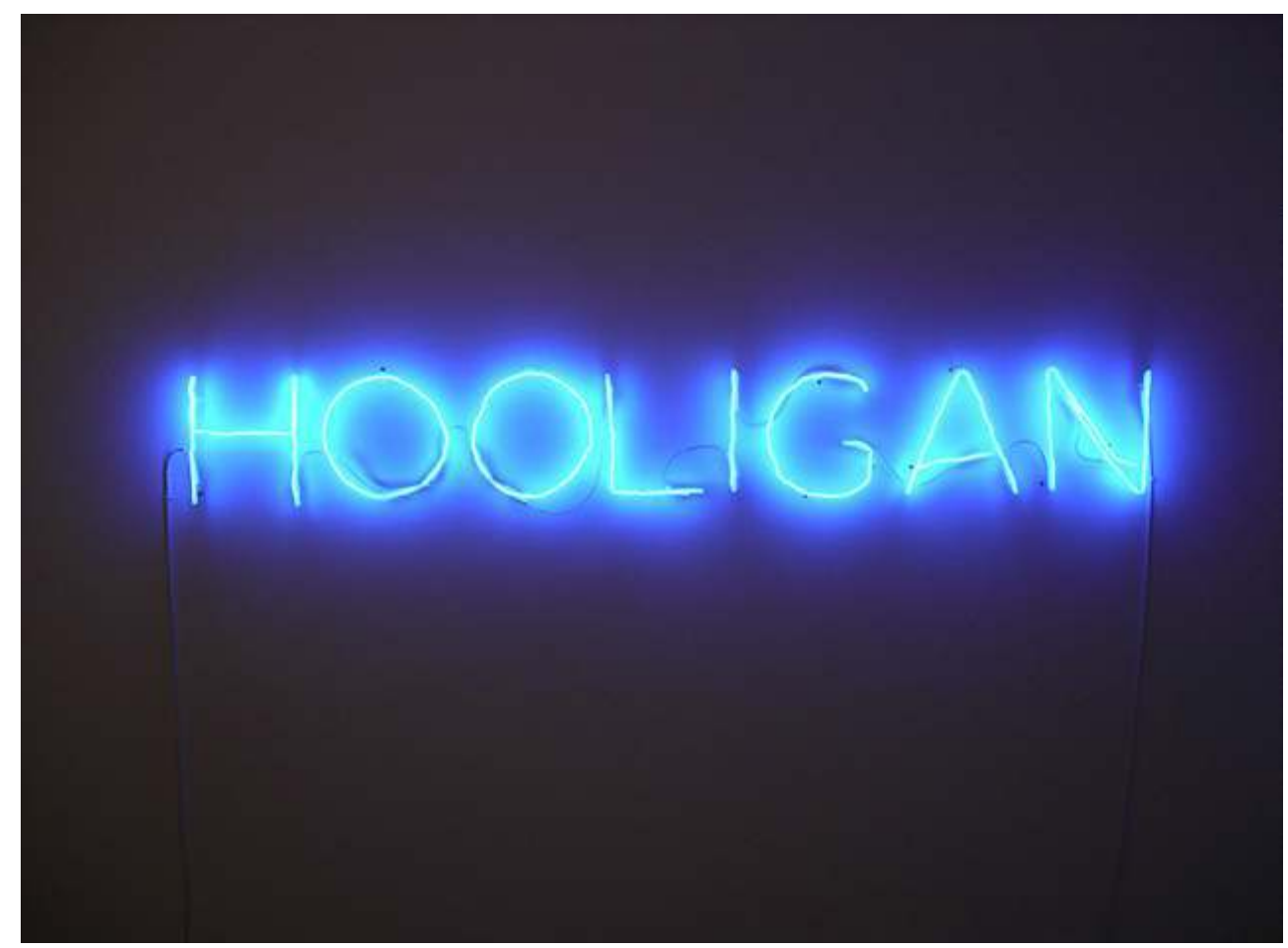

(c) ADAGP, Paris 2017. Courtesy the artist and kamel mennour, Paris/London 
À partir de ce parti pris muséographique, comment avez-vous réunis les objets présentés ?

Nous avions envie de faire quelque chose d'important concernant le "supportérisme". Mais, dès le début, nous sommes partis du constat que les objets des supporters n'étaient pas forcément conservés dans les musées. Au musée national du Sport, ou ailleurs en France, les objets de supporters généralement conservés sont des écharpes ou des fa-

nions, c'est-à-dire des objets qui représentent plus les spectateurs que les supporters ultras par exemple. Des enquêtes-collectes sur le "supportérisme" nous ont permis de recueillir beaucoup de données et comprendre et imaginer ce qui pouvait être réalisé en termes muséographiques.

Vous étiez surtout intéressés par le mouvement "ultras".

Tout ce qui est lié au mouvement ultras, qui forme une vraie contre-culture, a complétement échappé aux collections patrimoniales. Et c'est vraiment ce qui nous intéressait car, justement, cette contre-culture, cette sous-culture permet de dire beaucoup de choses sur la culture mainstream ou globale dans laquelle nous vivons. Donc, dès le début, nous avons voulu nous intéresser à ces groupes de supporters, les comprendre et savoir comment ils fonctionnent. Nous avons donc mis en place des enquêtes-collectes, comme celles qui existaient au musée des Arts et Traditions populaires depuis 1937 et que le Mucem continue à organiser depuis sa réouverture. Nous sommes partis - aidés par un chercheur spécialiste du sujet, Sébastien Louis, et un photographe, Giovanni Ambrosio - à la rencontre des groupes de supporters pour collecter des objets sur place ou susciter des dons. Pendant quatre ans, nous avons réalisé des enquêtes-collectes dans dix pays de la zone Euro Méditerranéenne, collecté près de 600 objets, 3000 photographies et plusieurs heures d'enregistrement.

\section{Quand est apparu ce mouvement en France?}

Le premier groupe d'ultras, le Commando Ultra, est né à Marseille, en 1984. Ce groupe nous a prêté une caisse en métal, une cantine toute cabossée, rouillée. Au musée, les régisseurs nous ont demandé pourquoi nous voulions exposer cet objet complétement "pourri", qui ne correspond en rien aux codes d'un musée classique ou de beaux-arts. Et, en même temps, nous avons mis six ou sept mois pour expliquer aux ultras que nous souhaitions cet objet. Ils ne voulaient pas s'en détacher, car il était très important à leurs yeux, cela fait quinze ans que cette cantine est utilisée lors des déplacements et cela lui donne beaucoup de valeur pour celles et ceux qui l'utilisent. Nous leur avons acheté une cantine neuve qu'ils utiliseront pendant les quatre mois de l'exposition.

\section{Et cette cantine a trouvé sa place?}

Présentée dans l'exposition, elle permet aux visiteurs de mieux comprendre le phénomène ultra. Parmi les préjugés, les supporters sont souvent associés à des gens violents ou alcooliques. En exposant cette caisse, nous racontons son histoire, son intérêt, et du coup l'importance qu'elle représente pour les supporters. Nous exposons aussi un album-photo pour montrer que les premiers ultra à Marseille étaient des minots de 15 ans qui avaient juste envie de faire comme en Italie, d'avoir des drapeaux et de ne pas rester assis dans un stade. Nous montrons que, anthropologiquement, cela a du sens, qu'il y a eu des échanges culturels entre ces gamins. Des lettres s'échangeaient entre ultras italiens, serbes et marseillais dans les années 1980. Les visiteurs peuvent comprendre que les ultras ne sont pas des personnes violentes 
par définition, mais qu'il s'agit aussi d'une culture qui permet d'expliquer la société. Par exemple, si un mouvement ultra est né en 2008 en Tunisie, c'est parce qu'il offre un lieu d'expression publique. Si la violence existe dans les stades, c'est parce qu'elle existe d'abord dans la société.

Chambre d'un adolescent supporter, Mazaugues, 2017, Mucem

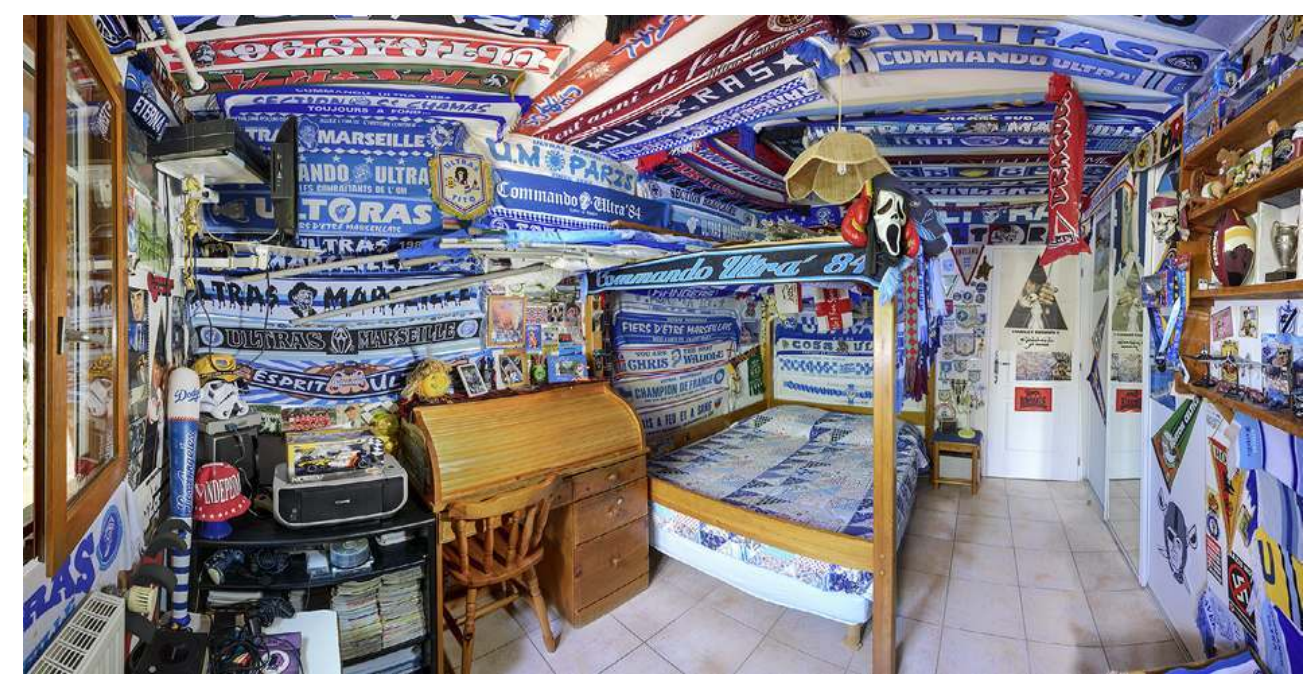

(c) Mucem/Yves Inchierman

\section{Certains objets ont-ils été intégrés aux collections?}

Pas encore. Nous avons inscrits les objets en matériel d'étude, nous avons ce statut depuis 2013 à notre portée et avons aujourd'hui cinq ans pour pouvoir les étudier et savoir ce qui est intéressant à garder ou pas dans les collections publiques. Et nous espérons vraiment faire entrer des objets à l'inventaire. Nous avons été en lien avec le projet de "grande collection Euro 2016" pour partager nos outils de recherche ; nous avons notamment travaillé avec eux pour mettre en commun nos outils pratiques, comme les fiches d'entretien, de description des objets ou d'autorisation de droit à l'image.

\section{Enfin, avez vous procédé à des achats ?}

La tête de la marionnette de Sepp Blatter a été difficile à acquérir. Nous avions vu cette marionnette dans les médias et il nous la fallait absolument, car il s'agit du seul objet matériel nous permettant de parler de l'économie. Cette marionnette a été réalisée en mai 2015 par une ONG, Avaaz, pour demander la démission de Sepp Blatter devant les locaux de la Fifa à Zürich lors de son 65 e congrès, et l'arrêt de l'esclavage moderne au Qatar sur les chantiers des stades de la Coupe du Monde 2022. Ensuite, les acquisitions comme celles des maillots se sont faites au fur et à mesure de l'avancée du projet.

Vous parlez de contraste, vous présentez aussi des objets de valeur?

Nous présentons la Coupe du Monde, la vraie, celle gagnée par la France en 1998, c'est un prêt de la fédération française de football. Et, à partir de décembre, nous avons pu exposer la Ligue des Champions, prêtée par l'UEFA, c'est une chance incroyable. Pour certaines personnes, la Coupe du Monde a trois fois plus de valeur que les toiles de Picasso que nous présentions l'an dernier, dont la valeur d'assurance est bien 
supérieure. Et ce n'est pas risible, c'est tout à fait compréhensible et il faut le prendre en compte.

Expliquer ce changement de référentiel vous permet de mieux faire comprendre la culture ultra.

En effet, l'importance et la valeur des objets peuvent être ailleurs. Les objets disent beaucoup des gens qui nous les ont prêtés. Par exemple, nous présentons un objet appartenant à un groupe d'ultras de Latina, une petite ville en dessous de Rome dans la province du Lazio, en Italie. Ce groupe nous a prêté une caisse en bois avec des klaxons, un objet extrêmement important pour lui. Nous avons découvert cette caisse dans leur local, pleine de poussière et avons dû négocier plusieurs heures avec eux pour le ramener à Marseille. Ils nous l'ont prêtée, mais avaient peur que nous la conservions mal. L'intérêt porté à cet objet par ce groupe est vraiment révélateur de la culture ultra. Et c'est une démarche importante pour nous, musée, de permettre de comprendre pourquoi la relation à cet objet intéresse aussi les autres, et appartient à la culture commune et au patrimoine. Nous avons beaucoup travaillé avec cette démarche et, au bout d'un moment, des ultras nous ont donné des choses en disant, "ok j'ai compris, effectivement toutes ces archives vont intéresser d'autres personnes qui n'y connaissent rien. Et vous permettre d'avoir un autre regard sur ce que nous faisons".

Toujours avec l'idée de dépasser les préjugés.

Dans les enquêtes-collectes, beaucoup de personnes nous ont dit, "je suis marié,j'ai des enfants, je les aime, mais le club de football passe avant ma famille". Nous ne pouvons pas faire l'impasse sur de telles passions. C'est ce que Christian Bromberger appelle des "passions ordinaires". C'est aussi ce que nous souhaitions faire en nous intéressant au monde ultras, prendre cette culture au sérieux et l'exposer telle qu'elle se définit.

Vous vous appuyez également sur des recherches récentes en sociologie, sur la spatialisation des supporters dans le stade

Nous sommes partis de la cartographie du vélodrome réalisée par Christian Bromberger en 1987. Avec des étudiants en licence 3 d'anthropologie, nous avons repris son questionnaire, en ajoutant ou en remaniant quelques questions. Les cartes présentées ont plus une valeur illustrative que statistique, car nous n'avons pu interroger que 300 personnes, sur un public de 25000 personnes pour le match en question, sachant que la capacité du stade est de 80000 personnes. De manière illustrative, ces cartes fonctionnent bien, elles montrent d'une part le lien entre la provenance géographique des spectateurs et leur place dans le stade. Et, d'autre part, le lien entre les catégories socio-professionnelles et les positions dans le stade.

Le football cristallise des contradictions par forcément évidentes à présenter.

La Fifa défend la paix par le football. Jules Rimet a justement créé la Fifa pour cet idéal. Sauf que, sous couvert de cette idéologie de paix, des millions d'euros peuvent être détournés ou des ouvriers migrants peuvent être détenus dans des conditions de travail effroyables sur les chantiers de la Coupe du Monde. C'est une grande contradiction de nos sociétés libérales, c'est aussi le cas des entreprises privées d'équipementiers sportifs qui parfois ont une image de marque liée à la solidarité et utilisent de la main d'œuvre mineure au Pakistan ou en Asie du Sud-Est. Nous sommes dans cette contradiction et c'est la raison pour laquelle il n'est pas évident d'en parler. Nous critiquons les méfaits de la société libérale contemporaine et, en même temps, nous achetons la dernière paire de Nike. Nous sommes tous dans cette négociation de nos identités avec ces contradictions. Dans l'exposition, nous avons 
voulu casser un peu certains préjugés pour pouvoir en parler et faire en sorte que le musée soit un lieu de réflexion sociale publique. J'enfonce des portes ouvertes mais, comme ce musée fonctionne avec de l'argent public et les impôts de tout le monde, nous invitons toutes les personnes qui le souhaitent à venir discuter de la place du football dans la société de manière citoyenne.

L'exposition commence et finit par le jeu.

Nous souhaitons finir de manière positive avec une dernière phrase : "et si le football redevenait citoyen ?". Cette exposition a été conçue comme une métaphore de la vie en société. Il ne s'agit pas d'une exposition sur le football, mais de société à travers le football. C'est pourquoi nous l'avons appelée Nous sommes football, avec l'idée que si nous voulions changer la société, nous avons tous une responsabilité. Et, de la même manière, que si nous avions envie de changer le football, nous avons tous et toutes notre part de responsabilité, en tant que citoyens, nous sommes tous et toutes impliqués dans cette histoire. Dès le début de l'exposition, la première salle permet de rappeler cette métaphore du jeu citoyen. La phrase d'Albert Camus rappelle des valeurs qui existent dans le football, la moralité notamment ${ }^{2}$. Les balles de peu évoquent le fait que le football est un jeu, c'est d'abord des gamins qui jouent dans des rues. Et, à la fin, pour boucler la boucle, nous sommes revenus sur l'initiative sociale forte de l'équipe d'Alma de Africa, une équipe de migrants espagnols. La corruption, le fric, les équipementiers sportifs, le dopage cela existe, mais il ne faut pas oublier que c'est quand même un jeu qui est pratiqué par des gamins et que le football peut avoir une portée citoyenne et sociale.

\section{En termes de publics, vous pensez avoir atteint vos objectifs?}

En octobre 2017, 30000 personnes avaient déjà vu l'exposition. Un matin de cette semaine j'ai pu faire des visites avec des supporters, des personnes qui m'ont dit qu'ils n'avaient jamais mis un pied dans un musée. Et, le soir, avec d'autres personnes issues d'un petit groupe de patrons d'entreprises locales qui avaient le même intérêt, la même passion pour cette exposition. Cela nous a permis de nous dire que nous ne nous étions pas trompés.

Vous avez observé les réactions des supporters?

Nous pouvons les identifier, et ils semblent extrêmement intéressés. Mais ces observations restent subjectives, une étude de public par l'observatoire du Mucem est prévue. Les médiateurs en salle et à la billetterie nous disent qu'effectivement, nous observons un changement. Les personnes qui visitent cette exposition ne sont pas habituées à venir au musée, nous avons déjà l'impression d'avoir une plus grande diversité sociale. Mais il y a un effet inverse : des personnes viennent au musée dans l'optique d'acheter un billet et lorsqu'ils apprennent que l'exposition temporaire est consacrée au football, ils repartent.

Des partenariats avec des clubs de supporters ont été réalisés pour leur faciliter l'accès à l'exposition?

Des actions ont été faites avec des écoles de football, avec la Fédération Française de Football et les Mutuelles du Soleil, qui sont des mécènes de l'exposition. En octobre, une journée a été réservée à tous les minots des écoles de football de Marseille. Avec l'aide d'une personne en service civique, nous avons identifié une vingtaine de clubs, notamment dans les quartiers nord qui n'ont pas l'habitude de venir au musée. Dans la partie apprentissage, nous avons réalisé un mur de photographies avec 500 enfants 
de ces clubs, et les avons invités à venir au musée. Avec les supporters, nous n'avons pas mis en place de collaboration fixe. Nous les avons invités et leur avons proposé d'organiser des visites de groupes gratuites. Mais, ils répondent peu pour l'instant, les groupes de supporters sont d'abord organisés pour aller en déplacement et pour suivre le club, et c'est déjà énorme.

Et des personnes de l'Olympique de Marseille viennent?

Des personnes de l'olympique de Marseille sont venues, mais le club n'a pas voulu participer au projet, même si nous avons essayé de les impliquer dès le début. Sans faire une exposition sur ce club, il y a une grande cohérence avec le fait d'être à Marseille aussi, avec un club emblématique. C'est dommage de n'avoir pu travailler ensemble, mais nous le comprenons, car le club va bientôt ouvrir un musée bientôt pour ses supporters.

Pour le livre qui accompagne l'exposition, vous avez aussi fait travailler des écrivains.

L'idée était de poursuivre la réflexion entamée dans l'exposition dans le catalogue. Nous ne voulions pas faire un catalogue scientifique, parce que cette littérature existe déjà. En sollicitant des écrivains, nous voulions rapprocher littérature et football, de la même manière que nous avions voulu associer musée et football. Il existe une revue de littérature et de sport, qui s'appelle Desports qui a bien voulu réaliser un numéro hors-série en guise de catalogue de l'exposition. C'est un petit livre, de moins de 25 euros, qui permet de faire un complément à l'exposition et montrer un autre point de vue ${ }^{3}$. Nous avons repris les trois grandes parties, et les sous parties, de l'exposition, pour réaliser le sommaire du catalogue. Et puis nous avons demandé à des journalistes sportifs comme Vincent Duluc et des romanciers, comme Yasmina Khadra ou Kaouther Adimi, d'exprimer avec leurs mots les relations qui les unissaient avec le football. Nous avons aussi des textes d'écrivains célèbres, comme Gabriel Garcia Marquez ou Eduardo Galeano. Nous avons voulu également faire un livre manifeste, que nous avons sous-titré Pour un football populaire. À la fin de l'ouvrage, nous proposons aux fédérations et, de manière plus générale, aux instances du football, d'évoluer vers un football citoyen.

\section{NOTES}

1. Le musée de l'innocence (Masumiyet Müzesi) a été créé à Istanbul en accord avec le roman éponyme de Orhan Pamuk. Le musée et le roman évoquent l'histoire de deux familles d'Istanbul. En 2014, ce musée a reçu le prix du musée européen.

2. "Ce que je sais de la morale, c'est au football que je le dois...", Albert Camus, 23 octobre 1957.

3. Nous sommes Foot. Pour un football populaire. Desport, Mucem, 2017. 


\section{RÉSUMÉS}

Avec Nous sommes Foot (du 11 octobre 2017 au 4 février 2018),le musée de société qu'est le Mucem souhaite rendre compte de la complexité que révèle le football en explorant les rives sud et nord de la Méditerranée, leur histoire et leur actualité.

INDEX

Mots-clés : exposition, sport, société

\section{AUTEURS}

\section{FLORENT MOLLE}

conservateur du patrimoine au Mucem, responsable des collections sport et santé et commissaire de l'exposition

\section{SAMUEL CORDIER}

directeur de l'Ocim 